Military Technical College

Kobry El-Kobba

Cairo, Egypt

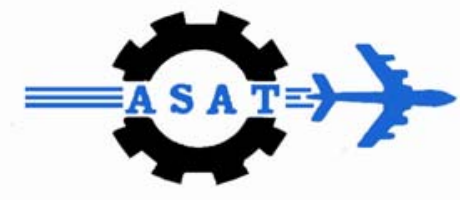

12-th International Conference

on

Aerospace Sciences \&

Aviation Technology

\title{
A SPACE-TIME ADAPTIVE PROCESSING WITH CHAOTIC AND QUADRATIC CHANGE OF PRF
}

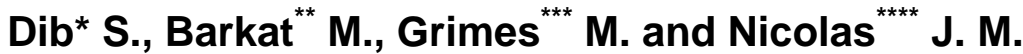

\begin{abstract}
In this paper, the effect of the change of Pulse Repetition Frequency (PRF) on the performances of the STAP with reduced rank is discussed. The numerical evaluation is based on two different models of changing the PRF, namely quadratic and chaotic but with two methods of reduction of the rank: Principle Components (PC) and Signal to Interference Noise Ratio (SINR metric). Rank reduction reduces the computing time and cost. The simulation results show that the change of PRF solves well the problem of ambiguities while using a reduced rank of covariance matrix of the environment. Indeed, the notches of the clutter are eliminated. Also, a comparative study between the two methods, quadratic change and chaotic change, shows that the chaotic change is better and this is due to the simplicity of the generation of the impulses on one hand, while on the other hand, the chaotic change has the possibility of taking a large variety of PRFs at the same time. In fact, we use the properties of a chaotic system, such as sensitivity of initial conditions, mixture, and density of periodic points to sweep all the space. The changes of frequencies used are numerous, and are generated (according to an equation modelling the environment).
\end{abstract}

\section{Key words}

Space time adaptive processing, STAP, PRF, chaotic change, quadratic change

\footnotetext{
* Assistant professor, Dpt. of electronics, University of Jijel, Algeria. Now she is a visiting scholar at the Dpt. of signal and image processing, ENST/TSI, Telecom Paris, France.

** Professor, Dpt. of Electrical Engineering, American University of Sharjah, UAE

*** Graduate student, Dpt. of Electronics, University of Jijel, Algeria.

${ }^{* * * *}$ Professor, Dpt. of signal and image processing, ENST/TSI, Telecom Paris, France.
} 


\section{INTRODUCTION}

In ground radars, all the ground returns are received with a Doppler frequency almost null. However, in airborne radars, the returns present a wide spectrum of Doppler frequencies because of the platform in motion. Space-Time Adaptive Processing, STAP, improves the capacity of radars to detect slow moving targets which can be masked by clutter or a jammer. In [1], we studied the STAP with full and reduced rank of the covariance matrix while introducing the concept of changing the PRF which showed its effectiveness for the elimination of the ambiguous notches of the clutter. It was proven in [1-8] that STAP is of a great utility for the detection of targets in the presence of interfering signals. It is an optimum tool for the design of air and space borne MTI radar. STAP has the unique property of compensating for the Doppler spread induced by the platform motion and thus, making the detection of slow targets possible.

In [8], Klemm considered the effect of a staggered PRF on the STAP performance. The numerical evaluation was based on two different stagger patterns: pseudo-random and quadratic. It was shown that quadratic staggering is equivalent to a constant acceleration of the radar platform. In case of perturbations of the platform motion, the precise knowledge of the perturbation is required to avoid mismatch between the target echo sequence and the Doppler filter.

In this paper, we propose the use of a chaotic change of the PRF and we compare the performances with those of the quadratic one. We proceed as follows: first, we present in Section 2 the structure of the covariance matrix to describe the environment in which the radar operates. In Section 3, we give a brief description of STAP with full and reduced rank, and of the improvement factor (IF), which is the parameter for detection performances. In Section 4, the proposed contribution in the change of PRF using both the quadratic and chaotic methods is presented. Then, our results along with a discussion are presented in Section 5.

\section{STRUCTURE OF THE COVARIANCE MATRIX}

The structure of the covariance matrix is given by [2]

$$
R=E\left[n n^{H}\right]=R_{c}+R_{j}+R_{n}
$$

where, $\boldsymbol{R}_{c}, \boldsymbol{R}_{j}$ and $\boldsymbol{R}_{n}$ are the covariance matrices of the clutter, jammers and thermal noise, respectively, such that

$$
\begin{aligned}
& \boldsymbol{R}_{c}=\sum_{k=1}^{N_{c}} \zeta_{k}\left(S_{t k} S_{t k}^{H}\right) \otimes\left(S_{s k} S_{s k}^{H}\right) \\
& \boldsymbol{R}_{j}=\sum_{i=1}^{N_{j}} \sum_{j=1}^{N_{j}} \alpha_{i} \alpha_{j}^{H} \otimes S_{s_{i}} S_{s_{j}}^{H}=A E A^{H}
\end{aligned}
$$

and 


$$
\boldsymbol{R}_{n}=\boldsymbol{E}\left[\boldsymbol{X}_{n} \boldsymbol{X}_{n}^{\boldsymbol{H}}\right]=\sigma^{2} \boldsymbol{I}_{J} \otimes \boldsymbol{I}_{K}=\sigma^{2} \boldsymbol{I}_{K J}
$$

The matrices $\boldsymbol{A}=\left[\boldsymbol{S}_{s_{1}}, \boldsymbol{S}_{s_{2}}, \ldots \ldots, \boldsymbol{S}_{s_{s_{j}}}\right]$ and $\boldsymbol{E}=\operatorname{diag}\left(\sigma^{2} \xi_{1}, \sigma^{2} \xi_{2}, \ldots \ldots ., \sigma^{2} \xi_{N_{j}}\right)$, with $\xi_{i}, i=1 \ldots \ldots . . N_{j}$, is the jammer to noise ratio (JNR), and $\zeta_{\mathrm{K}}$ is the clutter to noise ratio (CNR) per zone of clutter; $s_{t}$ and $s_{s}$ are, respectively, the directional temporal and space vectors.

\section{DESCRIPTION OF STAP}

\section{STAP with full rank}

The space-time adaptive processing with full rank for airborne radars is a continuation of the mono-dimensional adaptive processing [2]. The two-dimensional adaptive weights of the STAP will be calculated with each element and impulse. For the cell under test, the vector of data received can be expressed as

$$
X=\alpha S+X_{i}
$$

where, $\alpha$ represents a random amplitude, $S$ is the space-time vector of direction of the target, and $\boldsymbol{X}_{\boldsymbol{i}}$ is the vector of interferences. The output of the STAP is given by

$$
y=W^{H} X
$$

while the optimum weight (of the STAP), which maximizes the signal to interference noise ratio SINR, is obtained to be

$$
W_{\text {opt }}=\alpha \boldsymbol{R}_{i}^{-1} S
$$

$\boldsymbol{R}_{\mathrm{i}}$ is the covariance matrix of the ith interference which is supposed to be known. The performance of the processor can be discussed in terms of the Improvement Factor, IF. IF is defined as the ratio of the SINR of the output to that of the input of the Direct Form Processor, DFP. For an optimal processor, this factor is given by $[1,3]$

$$
I F_{\text {opt }}=S^{H} R_{i}^{-1} S \frac{\operatorname{tr}\left(R_{i}\right)}{S^{H} S}
$$

Note that a reversed peak of the clutter appears at the frequency in the direction of sight of the radar, and the width of this notch gives a measurement for the detection of slow moving targets.

\section{STAP with reduced rank}

The objective of the partially adaptive STAP is to reduce the complexity of the problem of adaptation, while carrying out always almost the same optimal performance. The partially adaptive algorithms of the STAP consists in transforming the data with a matrix $V \in C^{J k \times r}$ where $r \ll k J$. The weight vector of the reduced rank matrix is given by 


$$
W_{r}=R_{r}^{-1} S_{r}=\left(V^{H} R V\right)^{-1} V^{H} S
$$

where $R_{r}$ is the reduced covariance matrix of $X_{r}$. There are several methods of rank reduction [4-6], which differ in the shape of the processor and in the way the columns of the matrix are selected. The principal component is based on the eigenvectors conservation of the matrix of covariance of interferences corresponding to the dominant eigenvalues [4]. In the SINR metric method, the objective is to choose the $r$ columns of $V$ such that the loss in the performances of the SINR will be minimized. Berger and Welsh [6] chose the columns of $\boldsymbol{V}$ as being the eigenvectors of $\boldsymbol{R}$, which minimized the loss in the performance of the SINR. By taking into account the rank of the reduction matrix, the improvement factor of the reduced rank can be written as

$$
I F_{R R}=S^{H} V\left(V^{H} R V\right)^{-1} V^{H} S \frac{\operatorname{tr}\left(V^{H} R V\right)}{S^{H} V V^{H} S}
$$

\section{CHANGE OF PRF}

It is known that if the PRF is weak, Doppler ambiguities occur, and are caused by the overlapping of the edge lines with the true spectrum. This overlapping decreases gradually with each time the PRF is increased because the edge lines move away one from the other by leaving the true spectrum without a shift. So the idea of using the change of PRF appeared to solve the problem of Doppler ambiguities. We use two models of change for the PRF; namely, the quadratic change and the chaotic change. It should be noted that the change is done for an impulse to another (i.e., from pulse to pulse during processing).

\subsection{Quadratic Change of PRF [8]}

This type of change consists of increasing (or decreasing) the interval of repetition of impulses (PRI) in certain stages. Therefore, the PRI in the temporal frequency of the direction vectors of the target and clutter is multiplied by the term $(1+\varepsilon j / J)$ for each impulse $\mathrm{j}$. In practice, the best choice of $\varepsilon$, for the elimination of the ambiguous notches, is equal to 1 .

\subsection{Chaotic Change of PRF}

Traditionally, signals have been partitioned into two broadly defined classes; namely, deterministic signals and stochastic processes. This classification, however, overlooks another important class of signals, known as chaotic signals which share attributes with both deterministic signals and stochastic processes. Specifically, a chaotic signal has mostly a very irregular waveform, but it is generated by a deterministic mechanism [912]. Such a system must have the following properties. (i) It must be sensitive to the initial conditions, (ii) it must be topologically mixing, and (iii) its periodic orbits must be dense. We now describe each property. 


\section{Sensitivity to the initial conditions}

It means that if the starting state is changed, one expects that the general evolution of the system is also modified and the error will be of the same order as the signal itself. This can be seen clearly in Figure 1 , where we take as a reference value $x=0.1$ and introduce an error of 0.00001 . We can observe that the error changes the evolution of the results. Also, it has a value which approaches the one of the signal itself. Using the following dynamic system to generate the chaotic orbits $x(t)$, which are used for the generation of the PRF,

$$
\left\{\begin{array}{l}
x_{0} \in[0 ; 1] \\
x_{n+1}=f\left(x_{n}\right)
\end{array}, \text { with } f: x \rightarrow \operatorname{ax}(1-x)\right.
$$

the exhibitors of Lyapunov measure the rate of divergence of the close orbits. They are given by [9]

$$
\lambda\left(x_{0}\right)=\lim _{x \rightarrow \infty} \frac{1}{n} \sum_{k=1}^{n} \ln \left|f^{\prime}\left(x_{k-1}\right)\right|
$$

Using (8), Equation (9) becomes then

$$
\lambda\left(x_{0}\right)=\frac{1}{n} \sum_{k=1}^{n} \ln \left|a-2 a x_{n}\right|
$$

This expression of Lyapunov makes it possible to separate the zones where the system is chaotic from the zones where the system is stable as shown on Figure 2. In this case, if $\lambda\left(x_{0}\right)$ is negative or equal to zero, we are in the presence of a stable or periodic phenomenon; if not, it is chaotic.

\section{Capacity of mixture}

Topologically, mixing means that the system will evolve over time so that any given region or open set of its phase space it will eventually overlap with any other given region. If we consider two unspecified intervals $\mathrm{I}$ and $\mathrm{J}$ from $[0 ; 1]$, the first being regarded as source and the second as target, there exists an orbit whose first term $x_{0}$ is in I, and which has one of his elements $x_{n}$ in J. More precisely, the definition of the capacity of mixture is as follows:

$$
\forall] \alpha, \beta[,] \chi, \delta\left[\subset[0 ; 1], \exists x_{0} \in\right] \alpha, \beta\left[, \exists n \in N, x_{n} \in\right] \chi, \delta[
$$

We consider the function $f$ defined by Equation (8), with $a=4$. Let the interval of test be $[0 ; 1]$. If we subdivide this interval in ten equal parts, we obtains 10 sub intervals which are $[0 ; 0.1],[0.1 ; 0.2]$, and so on until the interval $[0.9 ; 10]$ and denoted $I_{1}, I_{2}, \ldots, I_{10}$. 
Consider the interval $[0.3 ; 0.4]$ as the starting interval and determine the final interval in which the values for this starting interval are.

As shown in Table1, the evolution of the final interval of $f(x)$ varies with the iterations since from a starting interval $[0.3 ; 0.4]$, the interval becomes $[0.024 ; 0.998]$ after 4 iterations. It should be noted that after one iteration only, sub intervals 9 and 10 are reached. The iteration produced points in sub intervals 2 to 6 and so on. We could have defined others sub-intervals for the interval $[0 ; 1]$ and still obtain similar results. Thus, a chaotic system will converge according to time (or from the iteration count) to others sub intervals in a manner at unforeseeable fact independently of the starting interval.

\section{Density of the periodic points}

By varying the parameter $a$, the following behaviour is observed. With a between 2 and 3 , the frequency oscillates around the value $(a-1) / a$ for some time and then it is stabilized at that same value. When $a$ is between 3 and 3.45 (approximately), the frequency oscillates between two values dependent of a but doest not stabilize. When a is between 3.45 and 3.54 (approximately), the frequency oscillates between four values and does not stabilize at any value. With a value of a slighter larger than 3.54 , the frequency will probably oscillate between 8 values, then 16,32 values, and so on. The lengths of the parameter intervals which yield the same number of oscillations decrease rapidly. This behavior is an example of a period-doubling cascade.

At $a=3.57$ (approximately), we have the onset of chaos at the end of the perioddoubling cascade. We can no longer see oscillations. Slight variations in the initial frequency yield dramatically different results over time, which is a prime characteristic of chaos. Most values beyond 3.57 exhibit chaotic behavior, but certain isolated values of a appear to show non-chaotic behaviour. These are sometimes called islands of stability.

For values of a greater than 4 , the values eventually leave the interval $[0,1]$ and diverge for almost all initial values. A bifurcation diagram, as shown in Figure 3, summarizes this. The horizontal axis shows the values of the parameter a while the vertical axis shows the possible long-term values of $x$. We can obtain the diagram of Feigenbaum from Equation (10) and we set for example $r=2.8$ until $r=4$. It is observed that from a principal branch, one passes to two branches which they are subdivided into two other ones (unfolding of the frequency).

As we approach 4 , we notice that the entire interval (from 0 to 1 ) is covered by this structure. The PRFs are generated according to the product: $P R F_{\max } x(n)$. The advantage of using a chaotic system to generate PRFs lies in:

(i) the simplicity of the generation of the impulses while basing itself on simple electronic circuits, such as the circuit of Chua, and

(ii) the possibility of taking a large variety of PRFs at the same time. In the case considered in this paper, we use a classical chaotic generator followed by a multiplier, so that we get all the PRFs in the interval $\left[0, P R F_{\max }\right]$. 


\section{RESULTS AND DISCUSSIONS}

The simulated radar is a linear network sidlooking (parallel with the longitudinal reference axis of the plane) of 8 antennas spaced by half the transmitted wavelength, with 10 impulses in the interval of coherent processing. The dimension of the adaptive process is thus $\mathrm{KxJ}=80$. The angle of elevation is fixed at $20^{\circ}$, and the angle of azimuth represents the only free parameter. The speed of the airborne radar is $100 \mathrm{~m} / \mathrm{s}$, and the frequency of transmission is $0.3 \mathrm{GHz}$. The environment of the interferences is composed of:

(i) Five jammers whose angles of azimuth are: $0^{\circ}, 180^{\circ}, 60^{\circ}, 90^{\circ}$ et, $72^{\circ}$, with jammer reports/ratios on noise (JNRs) of $13 \mathrm{~dB}, 12 \mathrm{~dB}, 11 \mathrm{~dB}, 10 \mathrm{~dB}$, and $9 \mathrm{~dB}$ respectively.

(ii) Clutter of ground covering the band $\left[-30^{\circ}, 30^{\circ}\right]$, and of clutter to noise ratio (CNR) equal to $8 \mathrm{~dB}$.

In Figure 4 we show the improvement factor (IF) versus the normalized Doppler frequency for the processor of direct form, DFP-PC, parameterized by rank $r$. We notice that if the rank is very small, the slow moving targets will be removed with the clutter, and thus will not be detected. We can then say that the rank should not be reduced below certain low values. The same observations are seen in the case of the SINR metric processor. In Figure 5, we present the improvement factor (IF) versus the normalized Doppler frequency for the processors DFP-optimal, DFP-PC and DFP-SINR metric respectively. We notice that the notches are thin and the slow moving targets are detected. We also observe ambiguous notches clutter with which the associated speeds are called blind speeds. The width of the notch did not change compared to that of the optimal processor. Thus, the reduction of the rank does not eliminate ambiguities from the clutter during the suppression of the noise, and that does not affect the detection of slow targets. The same observations are made when using the SINR metric method. Figure 6 shows the improvement factor (IF) for the three processors versus the standardized frequency Doppler, Ft, with the quadratic change of PRF. We note that the application of the change of the PRF removes the ambiguous notches clutter by leaving undulations in the bandwidth of the STAP filter for any values of the of PRF and number of ambiguous notches. The use of the change of PRF does not have any effect on the detection the slow targets. The same observations are made for a chaotic change, as shown in Figure 7, but we observe that the chaotic change associated the processor SINR metric gives performances which approach that of the optimal processor. This enables us to say that the chaotic change is preferred when using the quadratic change. Moreover, it is known that the generation of frequencies in a chaotic system is done using a simple circuit.

\section{CONCLUSION}

In this paper, we presented the fundamental principle of the STAP at reduced rank. We did the analysis using two methods: PC and SINR metric for a processor of direct form. The results showed that these methods allow a reduction in computing time and a reduction of the rank at low values without affecting the detection of slow moving targets. However, some ambiguous undulations or notches persist in the bandwidth. To eliminate the effect of these ambiguous notches on the detection performances, we used two methods of change of PRF; namely, quadratic and chaotic. We also showed that these two methods solve well the problem of ambiguities even with the reduction of 
the rank. Indeed, the notches of the clutter were removed. From the detection point of view, the two changes are good, but the chaotic one is preferred because of its simplicity of integration.

\section{REFERENCES}

[1] Dib, S., Barkat, M., Grimes, M. and Kioudj, H., " Analyse du STAP avec Valeurs Propres et Changement de PRF dans un radar aéroporté ॥, $2^{\text {ème }}$ Conférence sur le Génie Electrique CGE'02, EMP, Bordj El Bahri, Algérie, Dec 2002.

[2] Nguyen, H.N., « Robust Steering Vector Mismatch Techniques for reduced Rank Adaptive Array Signal Processing», PHD dissertation in Electrical and Computer Engineering, Virginia, Oct. 2002.

[3] Ward, J., "Space-time adaptive processing for airborne radar », Technical report of Technology, Lexington, MA, Dec 1994.

[4] Haimovitch, A.M., " An eigencanceler Adaptive radar by eigenanalysis methods», IEEE Transactions on Aerospace and Electronics Systems, Vol. 32, N², pp. 532542, Apr. 1996.

[5] Goldstein, J.S. and Reed, I.S., « Theory of partially adaptive radar», IEEE Transactions on Aerospace and Electronics Systems, Vol. 33, N4, pp. 1309-1325, Oct. 1997.

[6] Berger, S.D. and Welsh, B.M., « Selecting a reduced-rank transformation for STAP, a direct form perspective», IEEE Transactions on Aerospace and Electronics Systems, Vol. 35, N², pp. 722-729, Apr. 1999.

[7] Klemm, R., «Prospective in STAP research », Proceedings of the IEEE on Sensor array and multichannel Signal Processing. pp. 7-11 March 2000.

[8] Klemm, R., «STAP with staggered PRF», $5^{\text {th }}$ International Conference on Radar Systems, May 1991.

[9] Grimes, M., « Optimisation d'un Réseau de Télécommunication avec sécurisation», Thèse de Magister en Electronique, Université de Jijel, 2005.

[10] T.S. Parker, T.S. and Chua, L.O., "Chaos: a tutorial for engineers," Proc. IEEE, vol. 75, no. 8, pp. 982-1008, Aug. 1987.

[11] Haykin, S., "Chaotic signal processing: New research directions and novel applications", IEEE workshop on SSAP, Victoria, BC, Oct. 1992.

[12] Haykin, S., "Detection of signals in chaos", Proc. IEEE, vol. 83, no. 1, pp. 95-122, Jan. 1995. 
Table 1 Evolution of sub interval of $f(X)$ according to iteration count

\begin{tabular}{|c|c|c|c|c|c|c|c|c|c|c|c|}
\hline Iteration & Interval & $\mathrm{I}_{1}$ & $\mathrm{I}_{2}$ & $\mathrm{I}_{3}$ & $\mathrm{I}_{4}$ & $\mathrm{I}_{5}$ & $\mathrm{I}_{6}$ & $\mathrm{I}_{7}$ & $\mathrm{I}_{8}$ & $\mathrm{I}_{9}$ & $\mathrm{I}_{10}$ \\
\hline 0 & {$[0.3 ; 0.4]$} & & & & $\times$ & & & & & & \\
\hline 1 & {$[0.84 ; 0.96]$} & & & & & & & & & $\times$ & $\times$ \\
\hline 2 & {$[0.154 ; 0.538]$} & & $\times$ & $\times$ & $\times$ & $\times$ & $\times$ & & & & \\
\hline 3 & {$[0.521 ; 0.994]$} & & & & & & $\times$ & $\times$ & $\times$ & $\times$ & $\times$ \\
\hline 4 & {$[0.024 ; 0.998]$} & $\times$ & $\times$ & $\times$ & $\times$ & $\times$ & $\times$ & $\times$ & $\times$ & $\times$ & $\times$ \\
\hline
\end{tabular}
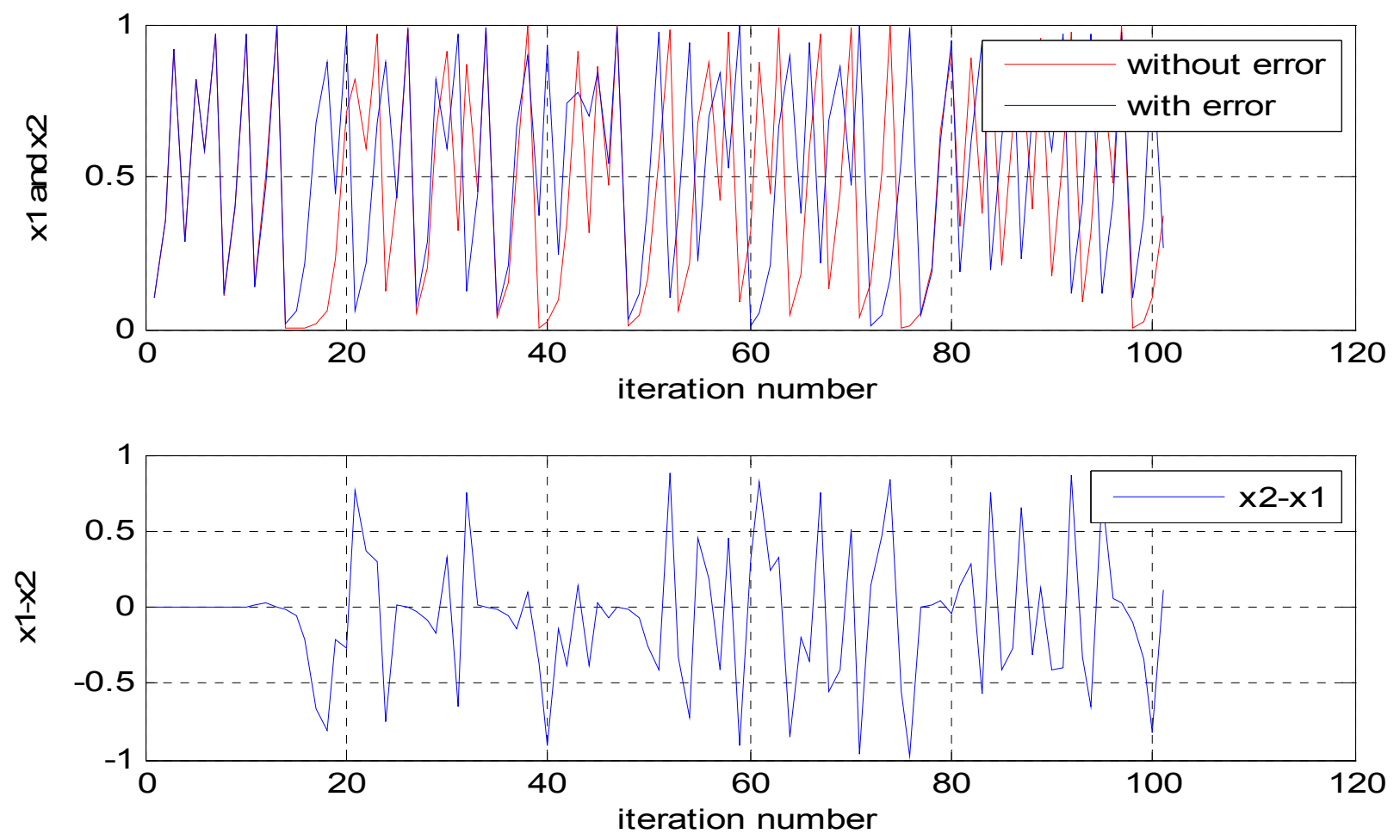

Fig.1 Measured error with an introduced error of 0.0001 


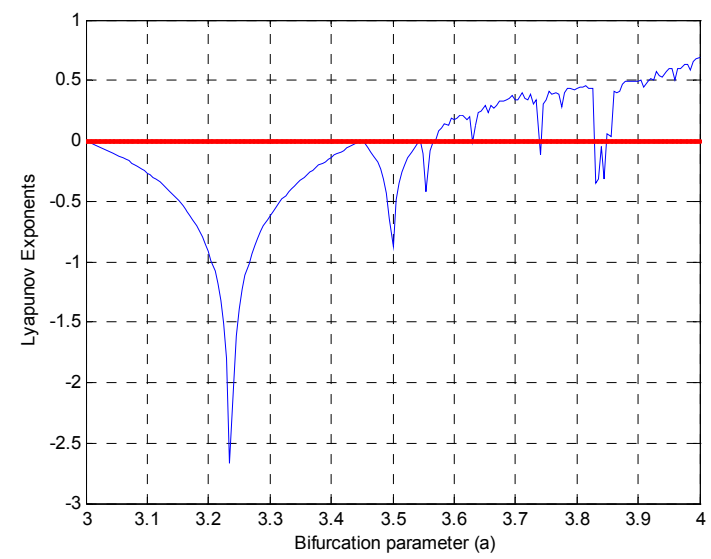

Fig.2 Curve of junction.

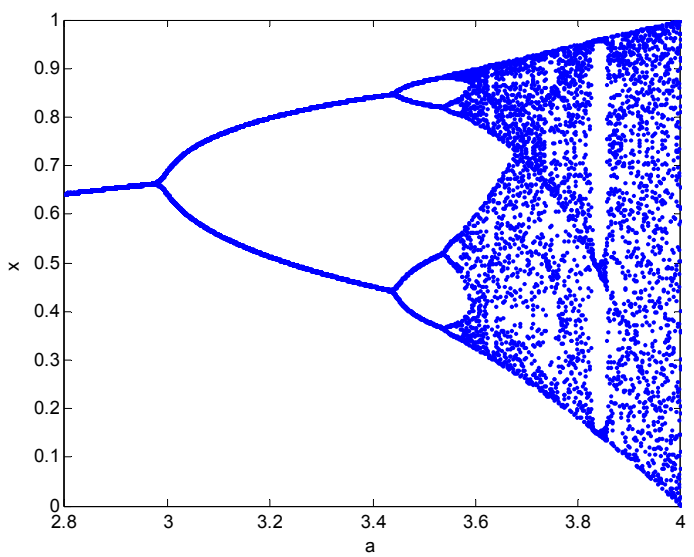

Fig.3 Chaotic effect to generate the PRF
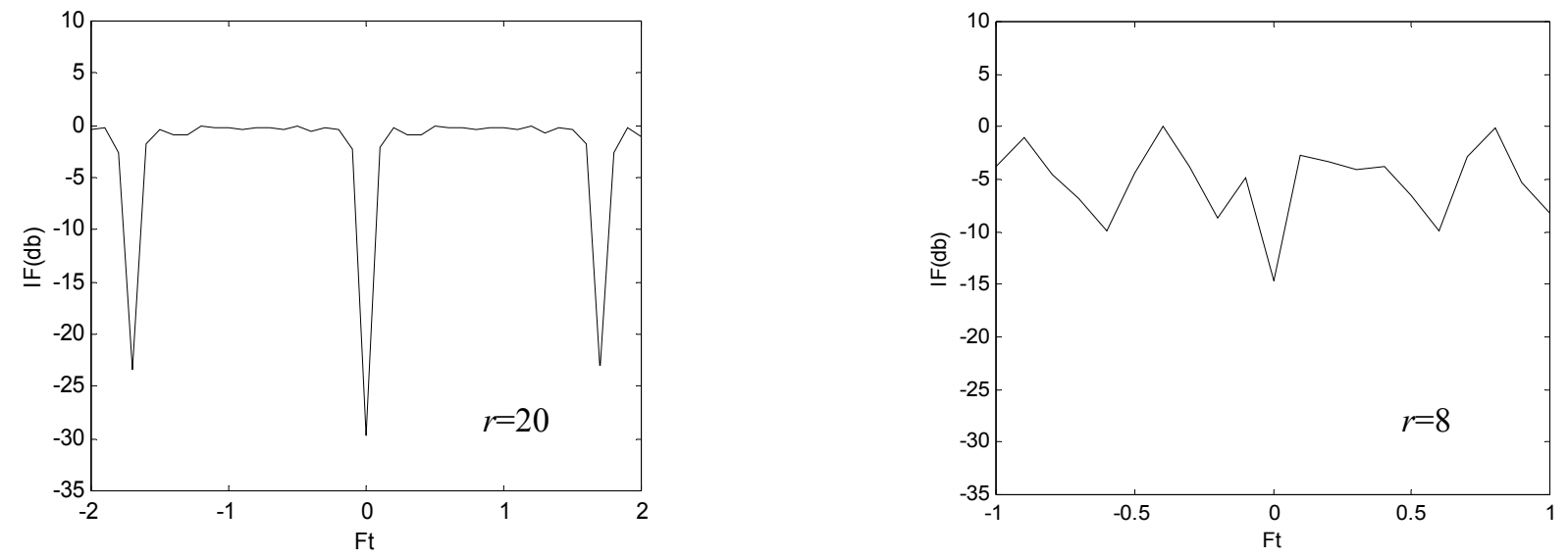

Fig. 4 Improvement Factor of the DFP-PC processor with reduced rank, $P R F=V R / 2 . \lambda$, $r=20$ and $r=8$. 
(a)

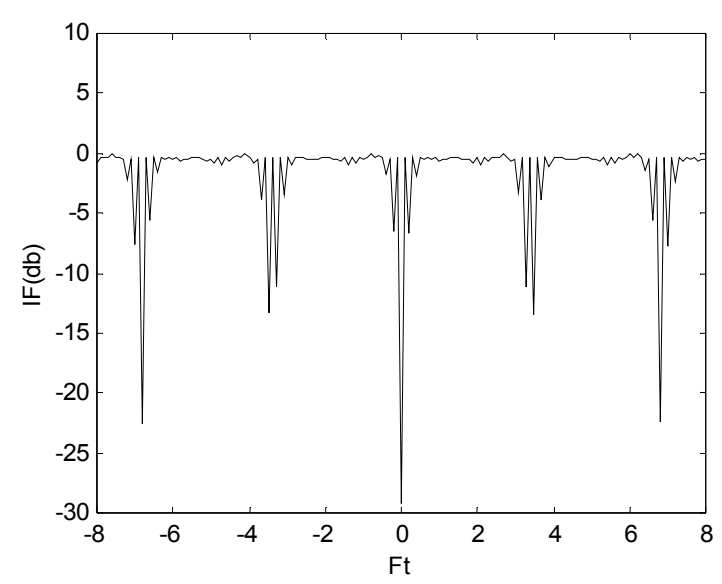

(b)

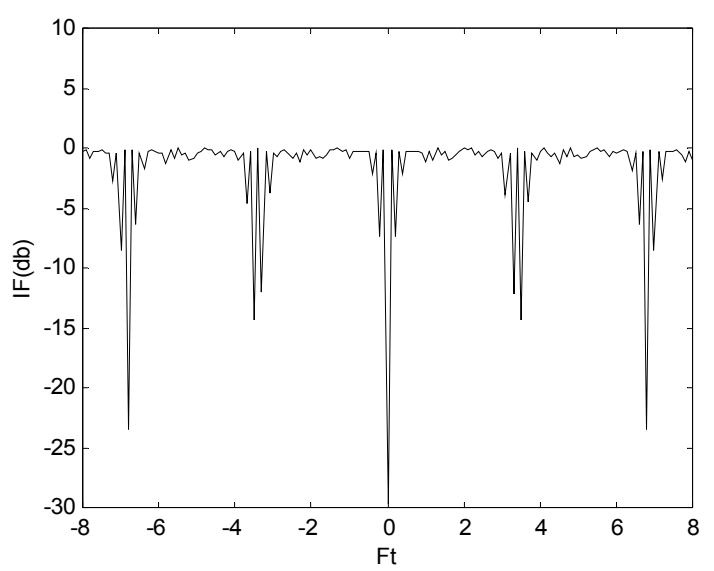

(c)

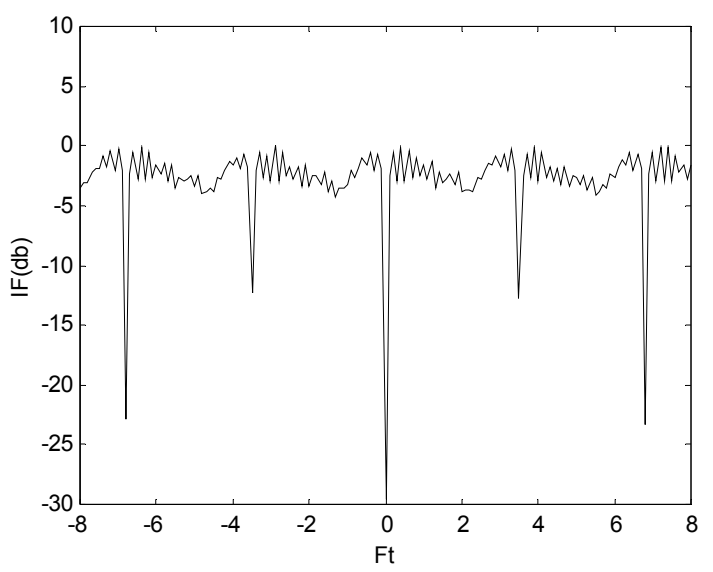

Fig. 5 Improvement Factor with constant $P R F, P R F=V R / 2 . \lambda, r=20$ :

(a) optimal processor, DFP (Direct Form Processor) ;

(b) the DFP-PC reduced rank processor and

(c) DFP-SINR metric reduced rank processor. 
(a)

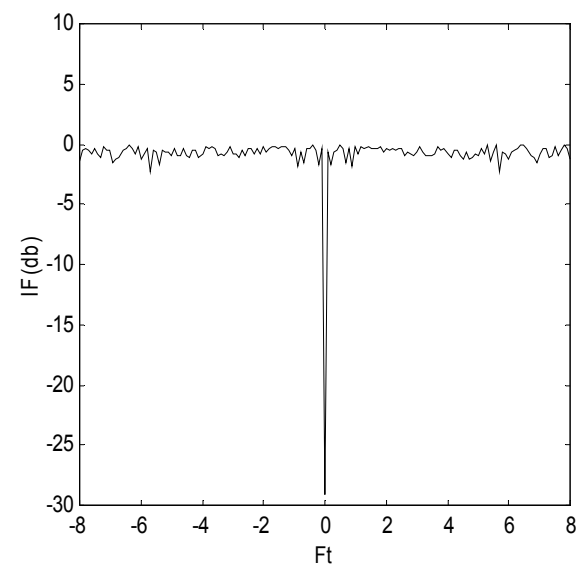

(b)

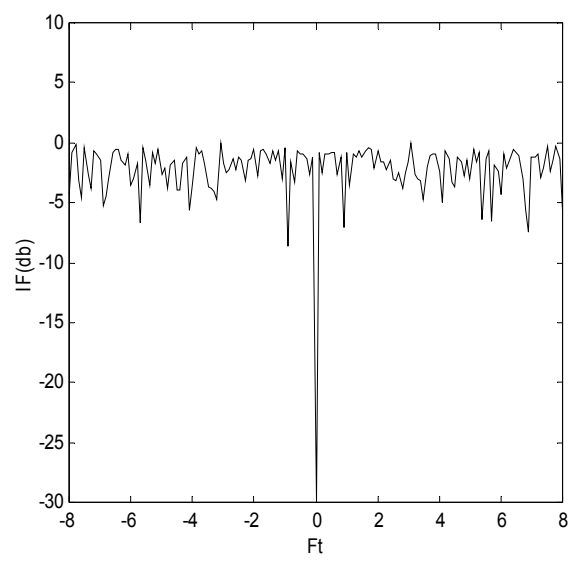

(c)

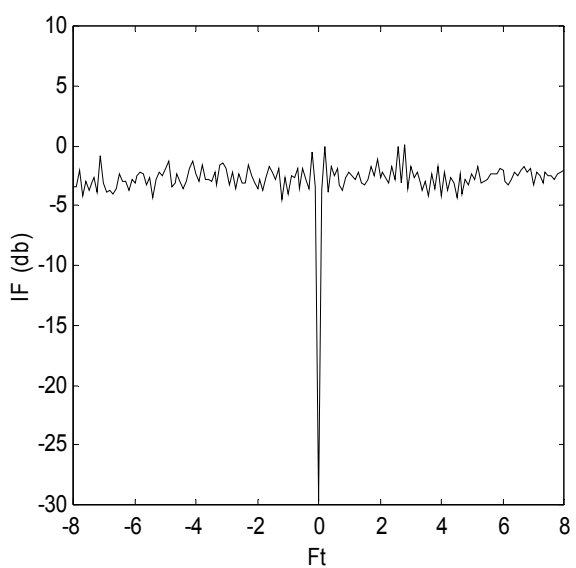

Fig. 6 Improvement Factor with quadratic change of $P R F, P R F=V R / 2 . \lambda, \varepsilon=1$, of :

(a) optimal processor, DFP (Direct Form Processor) ; (b) DFP-PC reduced rank processor and (c) DFP-SINR metric reduced rank processor.

(a)

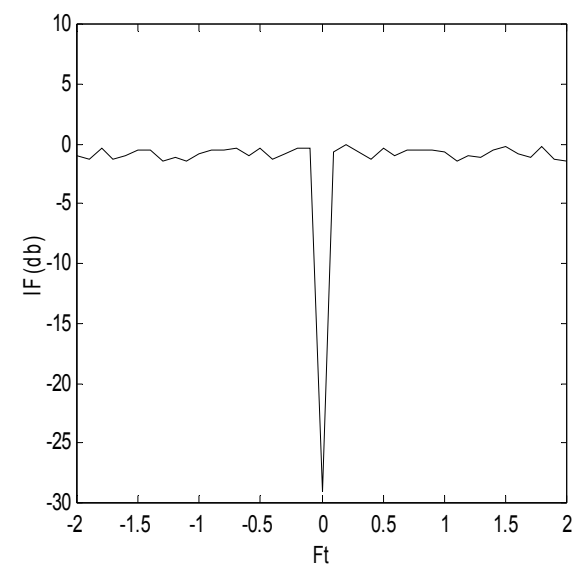

(b)

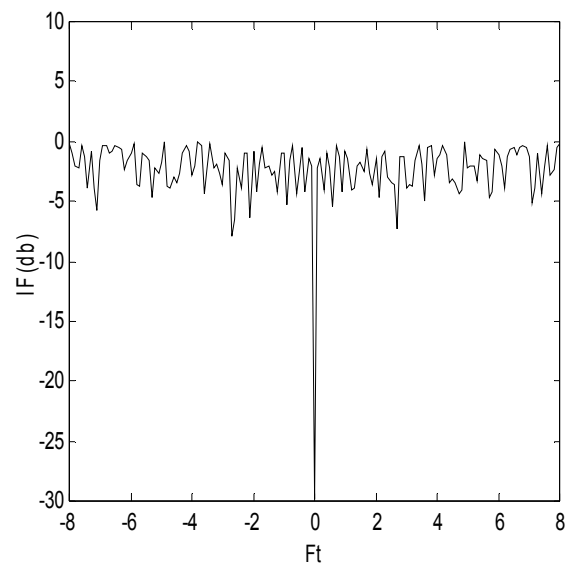

(c)

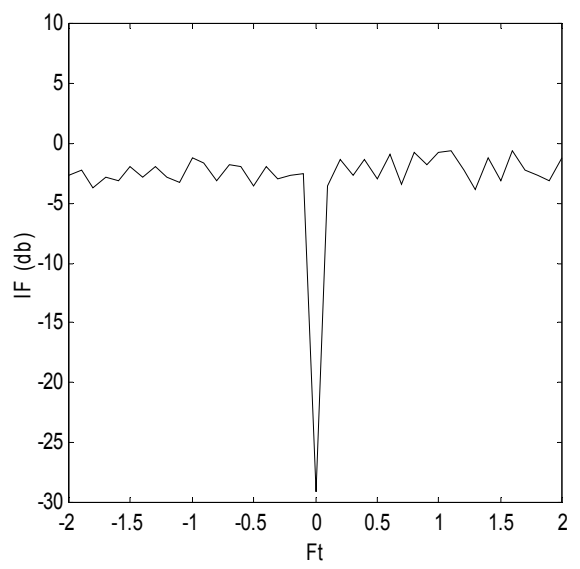

Fig. 7 Improvement Factor with chaotic change of $P R F, P R F=V R / 2 . \lambda, \varepsilon=1$, of : (a) optimal processor, DFP (Direct Form Processor) ; (b) DFP-PC reduced rank processor and (c) DFP-SINR metric reduced rank processor. 